\title{
What determines the radio polar brightening?
}

\author{
C. L. Selhorst ${ }^{1}$, A. V. R. Silva ${ }^{2}$, and J. E. R. Costa $^{1}$ \\ 1 CRAAM, Instituto Nacional de Pesquisas Espaciais, São José dos Campos, SP 12201-970, Brasil \\ e-mail: caius@craam.mackenzie.br \\ 2 CRAAM, Universidade Presbiteriana Mackenzie, São Paulo, SP 01302-907, Brasil
}

Received 17 March 2005 / Accepted 9 May 2005

\begin{abstract}
In order to explain the bright patches of emission near the poles of $17 \mathrm{GHz}$ solar maps, we have applied a previously developed atmospheric model based on radio observations. This 2-D model, which includes spicules, yields results in good agreement with brightness temperature values at the disk center, radius, and limb brightening $17 \mathrm{GHz}$ observations at equatorial and intermediate regions. Nevertheless, the intensity of discrete bright patches observed near the poles in $17 \mathrm{GHz}$ maps (as bright as $40 \%$ over the quiet Sun), can only be explained by holes in the spicule forest. These regions without spicules probably reflect the presence of polar faculae, which inhibit the appearance of spicules. Moreover, the absence of spicules over faculae explains the anti-correlation between the mean polar limb brightening and the solar cycle, because the polar faculae are known be anti-correlated with the solar cycle. Results from the model with spicule-less regions showed that: 1) for bare regions of the same size $\left(5^{\circ}\right)$, the limb brightening increases with the pole proximity; 2) larger regions yield more intense limb brightening; 3 ) the average height of the spicules greatly influences the solar radius; 4) the maximum excess brightness temperature of $40 \%$ is in very good agreement with the polar limb observations above bright patches.
\end{abstract}

Key words. Sun: atmosphere - Sun: radio radiation - Sun: fundamental parameters - Sun: faculae, plages

\section{Introduction}

In operation since 1992, the Nobeyama Radioheliograph (NoRH, Nakajima et al. 1994) has provided an excellent data base to study solar features at $17 \mathrm{GHz}$. An example is the prominent limb brightening seen in the daily solar maps. Shibasaki (1998) showed that this polar brightening consisted of extended bright areas and patches that are about $40 \%$ more intense than the $17 \mathrm{GHz}$ quiet Sun brightness. In Gelfreikh et al. (2002), the authors reported a spatial distribution of bright regions near the poles in $17 \mathrm{GHz}$ maps that resemble polar faculae. Selhorst et al. (2003) showed the existence of an anticorrelation between the mean polar limb brightening and the solar cycle. Moreover, the overall limb brightening distribution is not uniform, with the relative mean intensities greater near the poles $(25 \%)$ than in the equatorial $(15 \%)$ or intermediate regions $(10 \%)$.

In a previous work, Selhorst et al. (2005) developed an atmospheric model (hereafter referred to as the SSC model) in order to reproduce simultaneously three independent radio observations: the brightness temperature at disk center for frequencies ranging from 1.4 to $400 \mathrm{GHz}$, the observations of the radius and the limb brightening at $17 \mathrm{GHz}$. Moreover, the SSC is a 2-D model that takes into account the solar spherical curvature in order to study the solar radius and the limb brightening at $17 \mathrm{GHz}$. This model yields a radius of $\sim 970^{\prime \prime}$ at $1 \mathrm{AU}$ which is about $5^{\prime \prime}$ smaller than the mean observed value
(Selhorst et al. 2004). As for the limb brightening, we obtained $36 \%$ of center-to-limb variation, which is compatible with the larger values detected at the solar poles. However, it is larger than the brightening observed at equatorial and intermediate regions (Selhorst et al. 2003).

Several authors have suggested that spicules were responsible for a decrease in the limb brightening observed at radio wavelengths (Elzner 1976; Fürst et al. 1974; Braun \& Lindsey 1987). Thus, randomly distributed spicules were introduced in the SSC model. As a result of this heterogeneous atmosphere, the relative limb brightening was reduced to values of $12 \%$ above the quiet Sun. By varying randomly the physical parameters of spicules (such as density, temperature, height and inclination angle), the brightness temperature distribution across the disk agreed very well with observations at $17 \mathrm{GHz}$, except for the polar regions. Furthermore, the model has shown that the solar radius at $17 \mathrm{GHz}$ is mainly determined by the average height of the spicules, increasing as the spicule height increases.

In this work, we discuss how the SSC model with spicules can describe the polar limb brightening at $17 \mathrm{GHz}$ and the physical parameters involved in this solution. The next section summarizes the observations. In the third section we describe the SSC model with spicules and the implementation of regions without spicules. The comparison between the model and $17 \mathrm{GHz}$ polar observations is presented in Sect. 4. Discussion 
of the results and the main conclusions of this work are given in Sect. 5.

\section{Observations}

Daily solar maps from NoRH, with good spatial resolution (10-18"), from 1992 to 2004 were analyzed. This high spatial resolution enables the study of the limb brightening at $17 \mathrm{GHz}$, which has a large contribution from the bright patches observed near the poles, as can be seen in Fig. 1.

From the synthesized NoRH images, we have extracted the brightness temperature distribution profiles across the disk by taking radial scans that cross the center of the solar disk. Each cut provides a brightness temperature profile, where the brightening near the limb is quite evident, especially near the North Pole where the scan crosses a bright patch of emission (shown as a sharp increase on the right hand-side of the brightness profile in Fig. 1, bottom panel). The limb brightening has a nonuniform distribution as a function of latitude (Selhorst et al. 2003), with greater intensities at the poles mainly due to the presence of bright patches that reach brightness temperatures as high as $40 \%$ above the quiet Sun temperature. Because the polar brightening consists of many bright patches, frequently, the scan profiles may present different brightening in directly opposite limbs (as is the case of the profile shown in the bottom panel of Fig. 1).

\section{2-D atmospheric model with spicules}

\subsection{The SSC model}

The atmospheric model previously developed (Selhorst et al. 2005) consists of temperature and density (electron and proton) distributions as a function of height, from the photosphere up to $40000 \mathrm{~km}$ in the corona. The SSC model was developed as a bi-dimensional space reproducing one quadrant of the Sun. Two matrices were created representing the spatial distributions of temperature and the product of the electron and proton densities, $n_{\mathrm{e}} n_{\mathrm{p}}$. These matrices were divided into seven smaller matrices to facilitate computational handling, which were sequenced following the solar spherical curvature. The total angular region covered by the matrices is larger than one quadrant $\left(90^{\circ}\right)$, ranging from polar angles $-10^{\circ}$ through $100^{\circ}$ to include the ray tracing at the limb.

To calculate the brightness temperature as a function of position across the solar disk, the emission mechanism at $17 \mathrm{GHz}$ was assumed to be thermal bremsstrahlung. The radiative transport was calculated along paths represented by horizontal lines, separated by $1^{\prime \prime}$ from each other. Thus, a brightness temperature profile from the center to the solar limb was obtained with an excess brightening of $100 \%$ near the limb. Assuming the Sun to be symmetric, this profile was folded around the Sun center. Since the NoRH maps have a maximum spatial resolution of $10^{\prime \prime}$, the brightness temperature profile was convolved with a $10^{\prime \prime}$ FWHM Gaussian beam. Then, the convolved excess temperature at the limb decreased to $36 \%$ above disk center values, which is very close to the maximum limb brightening observed in the polar region. However, it is still higher than the
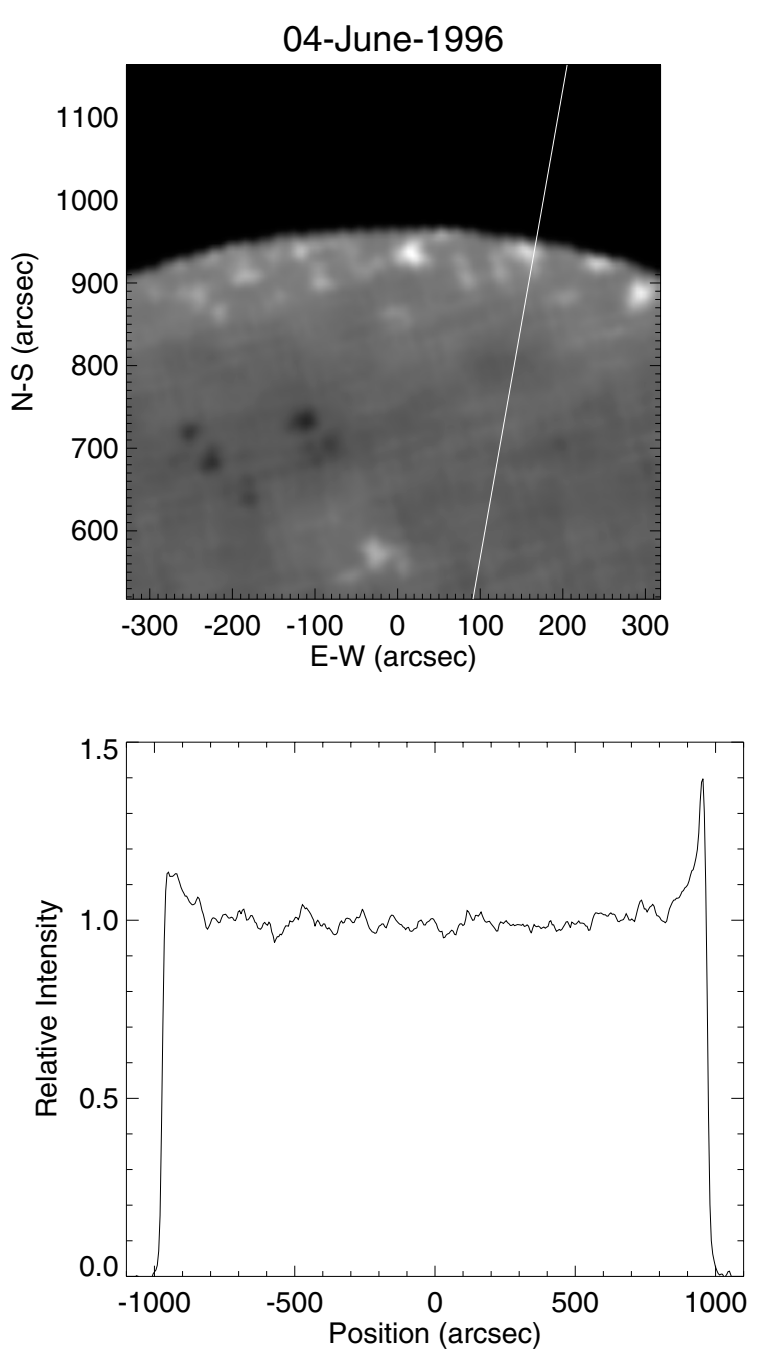

Fig. 1. Top: solar North pole of a $17 \mathrm{GHz}$ map during the solar minimum. The white straight line represents a scan in the map, which yields the intensity profile shown in the bottom figure. The scan crosses a bright patch very close to the limb and the pole, that causes a sharp increase in the intensity profile near the limb.

mean values observed in the equatorial (15\%) and intermediate regions $(10 \%)$.

To reduce even the further limb brightening and increase the solar radius at $17 \mathrm{GHz}$ (in order to fit the observations) the presence of spicules were included. In the model, spicules are represented as cylinders of constant density and plasma temperature with typical spicule composition. The spicules were randomly distributed around the limb in the SSC model, inclined with respect to the solar surface replacing the temperatures and densities of the local atmosphere. This procedure resulted in a solar radius closer to the observational values and a limb brightening compatible with the brightening observed at equatorial and intermediate regions.

\subsection{Model with spicule-less regions}

As mentioned before,however, the solar maps at $17 \mathrm{GHz}$ present limb brightening as high as $40 \%$ (Selhorst et al. 2003; Shibasaki 1998) above the quiet Sun values, that is 
Table 1. Spicule-less simulation results.

\begin{tabular}{ccc|cccc}
\hline \hline Figure & Spicule parameter & Spicule-less region & \multicolumn{4}{|c}{ Results } \\
\hline & $\begin{array}{c}\text { height } \\
(\mathrm{km})\end{array}$ & heliographic angle interval & center $T_{\mathrm{b}}$ & \multicolumn{2}{c}{ Limb bright. } & radius \\
& $5-7$ & $\left({ }^{\circ}\right)$ & $(\mathrm{K})$ & $(\%)$ & width $\left(^{\prime \prime}\right)$ & $\left({ }^{\prime \prime}\right)$ \\
\hline 3a & $5-7$ & $76.2-81.4$ & $10720 \pm 120$ & 21.4 & 55.4 & 972.4 \\
3b & $5-7$ & $78.5-83.7$ & $10670 \pm 100$ & 26.3 & 39.2 & 972.5 \\
3c & $5-7$ & $80.2-85.5$ & $10700 \pm 120$ & 30.0 & 17.4 & 972.4 \\
$3 \mathrm{~d}$ & $5-10$ & $80.2-90.0$ & $10740 \pm 100$ & 40.0 & 14.5 & 972.5 \\
$3 \mathrm{e}$ & & $80.2-90.0$ & $10800 \pm 100$ & 39.4 & 19.4 & 975.1 \\
\hline
\end{tabular}

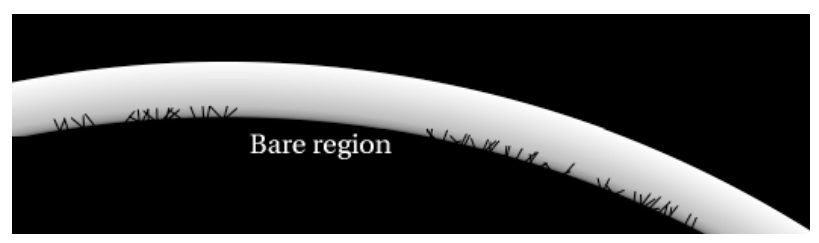

Fig. 2. Example of a bare region (region without spicules) near the pole, between 80 and $90^{\circ}$, in the temperature matrices.

not explained by the SSC model. Thus, a modification of the SSC model was needed in order to explain the $17 \mathrm{GHz}$ emission near the poles. Therefore, we suggest that the discrete bright polar patches observed in $17 \mathrm{GHz}$ maps are due to holes in the spicule forest right above polar faculae. Gaizauskas (1984) observed that there are no spicules over faculae as well as over plages (Zirin 1988). Moreover, faculae are known to be more abundant near the poles (Erofeev 2001; Unruh et al. 1999; Ortiz et al. 2002) and the radio polar bright regions are close to the polar faculae regions (Riehokainen et al. 1998; Gelfreikh et al. 2002).

To test this hypothesis, model simulations were performed with regions without spicules near the poles (Fig. 2). In all simulations, the spicules were $500 \mathrm{~km}$ wide, random temperatures from 7 to $13 \times 10^{3} \mathrm{~K}$, electron and ion densities ranging from 2 to $6 \times 10^{10} \mathrm{~cm}^{-3}$, and randomized inclination angles from 30 to $150^{\circ}$ (Sterling 2000; Zirin 1988, and references therein). The results of the simulations are listed in Table 1 and plotted in Fig. 3, each result represents a mean of 20-40 simulations with all spicules randomly generated around the limb. The first column shows the figure panel related to the simulations, the second column shows the height range of the spicules, and the heliographic angles of regions without spicules in the simulations are shown in the next column. The results are listed in the next four columns: the model brightness temperature at disk center, the limb brightening intensity and its width, and the resultant solar radius.

The first three simulations presented in Table $1(\mathrm{a}-\mathrm{c})$ have regions without spicules within a $5^{\circ}$ heliographic interval. However, the distances of these regions to the pole are different. These simulations show a higher limb brightening for spiculeless regions closer to the pole, whereas the limb brightening width decreases due to projection effects of the solar curvature. For example, the limb brightening intensity increases from 21.4 to $30.0 \%$ as the bare region approaches the pole (first and third simulations), whereas the width shows a larger abrupt decrease
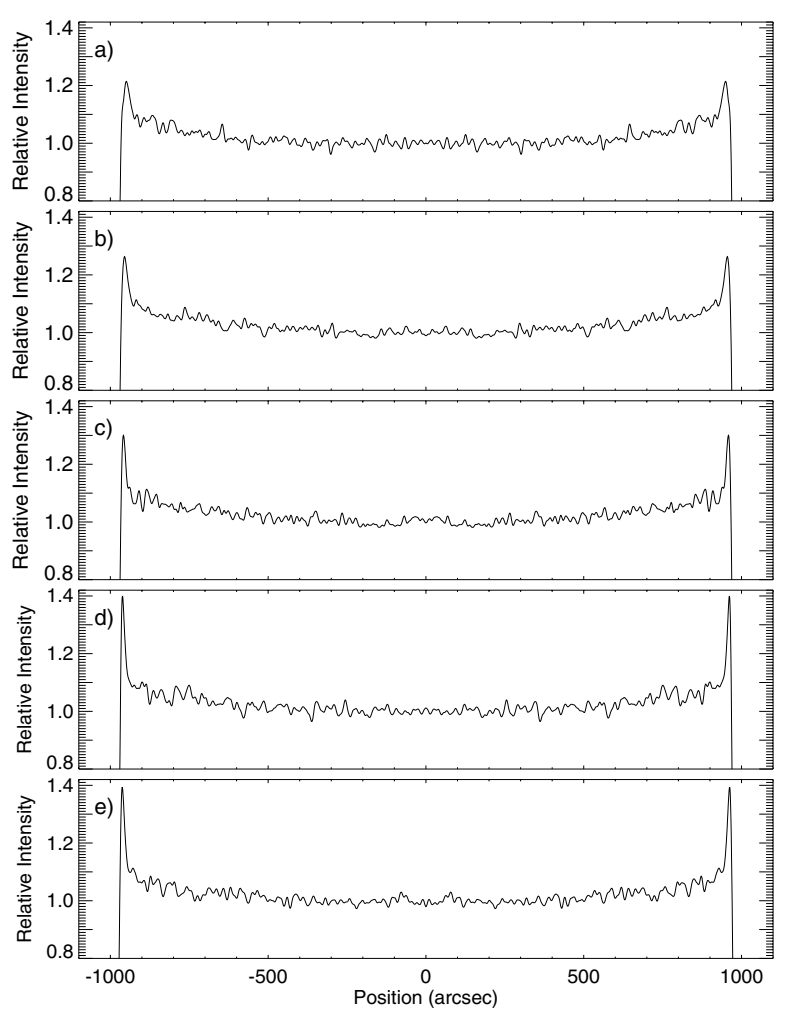

Fig. 3. Center-to-limb brightness temperature profile, calculated as the mean of a set of simulations using the SSC atmospheric model with regions without spicules. Each panel represents a bare region with different parameters, which are listed in Table 1.

from 55.4 to $17.4^{\prime \prime}$ in the same simulations. The increase in the max intensity of the limb brightening accompanied by a decrease in its width is clearly seen in the top three panels of Fig. 3.

In the last two simulations, the role of the spicule height was studied in the simulations with a spicule-less region. In these simulations, the area of the region without spicules was twice as large as those in the first three models of Table 1 . The results show a thin and intense limb brightening, that reached approximately $40 \%$ above the quiet Sun values in both simulations (Figs. 3d and 3e). These limb brightening values are larger than those obtained from the SSC model without spicules (36\%, see Selhorst et al. 2005). This result can be explained by spicules located beyond $90^{\circ}$, which are optically thick and emit more at $17 \mathrm{GHz}$ than the replaced local atmosphere. 
The very narrow limb brightening width is explained by the location of the bare region, between $80.2-90.0^{\circ}$. At this location the projected area is only about $15^{\prime \prime}$, which would be the apparent size of a bright region on a solar map. Moreover, the results yield a radius increase, which implies that the spicule height is more important for a radius increment than for limb brightening.

\section{Comparison between the model and the $17 \mathrm{GHz}$ polar observations}

The polar limb brightening observed at $17 \mathrm{GHz}$ is not homogeneous (Selhorst et al. 2003), moreover the observations show the existence of compact bright patches unevenly distributed around the limb. In this section we present the results of a comparison between the observations and simulations. The observation considered here is a scan close to the solar poles of a $17 \mathrm{GHz}$ solar map taken during the last solar minimum (June 4th, 1996). The scan crosses an intense bright patch in the North pole (Fig. 1), while the other pole presents the usual limb brightening of approximately $13 \%$ (Fig. 4, gray curve). The scan intensity was normalized to the quiet Sun brightness temperature (taken as the most common value of the map).

First, the brightness temperature calculated from the SSC model was obtained by varying randomly all physical parameters of the spicules (except the width which was fixed at $500 \mathrm{~km}$ ) which were distributed randomly throughout the limb. The spicules physical parameters were randomly chosen from ranges in temperatures (7000-13000 K), densities $\left(2-6 \times 10^{10} \mathrm{~cm}^{-3}\right)$, inclination angle $\left(30^{\circ}-150^{\circ}\right)$ and heights $(5000-7000 \mathrm{~km})$. The result for this simulation (Fig. $4 \mathrm{a}$ black line) was $14 \%$ of limb brightening that matches the observation of the Southern pole (left hand-side of Fig. 4a).

Nevertheless, to reproduce the $40 \%$ limb brightening intensity observed in the Northern pole, a bare region from 80.2 to $90.0^{\circ}$ heliocentric angles was included in the simulation. The physical parameters of the spicules outside this region were the same as in the previous simulation. The resulting brightness profile, taken as a mean of 27 simulations, the same as in Fig. 3d and Table 1, is shown as a dark solid line on the right of Fig. 4b. As can be seen from the figure, the model with a bare region represents very well the observed limb intensity $(40 \%$ above the quiet Sun), however, the limb brightening width is narrower than the observed one. A modification in the temperature distribution above this spicule-less region could increase this width.

\section{Discussion and conclusions}

The SSC atmospheric model developed by Selhorst et al. (2005) showed that spicules distributed randomly throughout the limb reduce the simulated limb brightening at $17 \mathrm{GHz}$ to values close to the mean values observed in equatorial $(15 \%)$ and intermediate regions $(10 \%)$. Nevertheless, there are discrete bright patches that can reach up to $40 \%$ above the quiet Sun brightness temperature, especially near the polar limb. It is known that there are no spicules over chromospheric plages (Shibata \& Suematsu 1982; Zirin 1988) and the same behavior
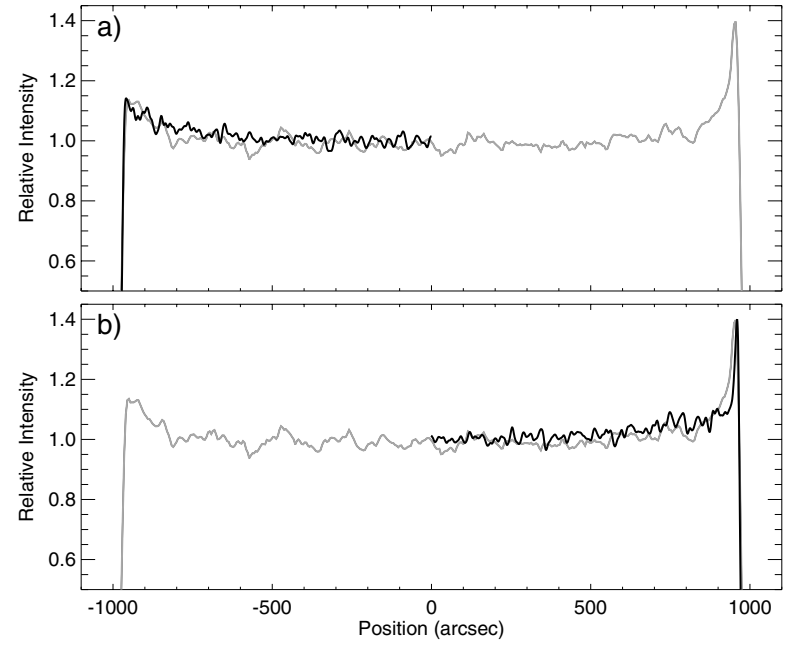

Fig. 4. Comparison between the observed $17 \mathrm{GHz}$ brightness temperature profile (gray curve) and a) the mean of SSC model simulations with spicules distributed throughout the limb and $\mathbf{b}$ ) with spicule-less regions close to the North pole.

was observed for faculae (Gaizauskas 1984). Moreover, polar faculae and polar limb brightening are both anti-correlated with the sunspot cycle (Efanov et al. 1980; Makarov \& Makarova 1996; Koshiishi 1996; Selhorst et al. 2003). Therefore, based on these observations, we suggest that holes in the spicule forest associated with faculae could yield patches of intense limb brightening near the solar poles.

To test this hypothesis, we have performed simulations containing regions without spicules near the poles at different positions and for 2 distinct sizes. These simulations with spiculeless regions have shown that:

1. for regions of $5^{\circ}$ in size, the limb brightening increases with the pole proximity;

2. larger region result in more intense limb brightening;

3. the average height of the spicules influences more the solar radius than the maximum of the limb brightening at $17 \mathrm{GHz}$;

4. the maximum excess brightness temperature obtained was $40 \%$, in very good agreement with the polar limb observations above bright patches.

Although the presence of spicules reduces the limb brightening at $17 \mathrm{GHz}$ to values close to those observed in solar equatorial and intermediate regions, the existence of small regions without spicules could produce intense patches of radio emission near the solar poles. These polar bare regions are probably associated with the presence of faculae in these region.

The model proposed here considers only the bremsstrahlung emission, which for distinct radio frequencies is produced in different heights of the solar atmosphere. The presence of spicules in the atmosphere changes the local physical parameters, such as temperatures and densities (electrons and ions), yielding different values for the brightness temperature. Thus, the resulting limb brightening will vary according to the radio wavelength. For example, at higher frequencies, the brightening at the solar limb could be reduced or even completely suppressed. 
To improve the model, changes in the local atmosphere (temperature and densities) caused by the presence of faculae should be made in order to provide a better agreement with observations.

Acknowledgements. We are grateful to the NoRH staff for obtaining the full disk $17 \mathrm{GHz}$ maps and making them available over the Internet. The Nobeyama Radioheliograph is operated by NAOJ/Nobeyama Solar Radio Observatory. C.L.S. acknowledges financial support from the Brazilian agency FAPESP, grant number 01/02106-3.

\section{References}

Braun, D., \& Lindsey, C. 1987, ApJ, 320, 898

Efanov, V. A., Moiseev, I. G., Nesterov, N. S., \& Stewart, R. T. 1980, in Radio Physics of the Sun, IAU Symp., 86, 141

Elzner, L. R. 1976, A\&A, 47, 9

Erofeev, D. V. 2001, Sol. Phys., 203, 9

Fürst, E., Hachenberg, O., \& Hirth, W. 1974, A\&A, 36, 123
Gaizauskas, V. 1984, Sol. Phys., 93, 257

Gelfreikh, G. B., Makarov, V. I., Tlatov, A. G., Riehokainen, A., \& Shibasaki, K. 2002, A\&A, 389, 618

Koshiishi, H. 1996, Ph.D. Thesis

Makarov, V. I., \& Makarova, V. V. 1996, Sol. Phys., 163, 267

Nakajima, H., Nishio, M., Enome, S., et al. 1994, Proc. IEEE, 82, 705

Ortiz, A., Solanki, S. K., Domingo, V., Fligge, M., \& Sanahuja, B. 2002, A\&A, 388, 1036

Riehokainen, A., Urpo, S., \& Valtaoja, E. 1998, A\&A, 333, 741

Selhorst, C. L., Silva, A. V. R., \& Costa, J. E. R. 2004, A\&A, 420, 1117

Selhorst, C. L., Silva, A. V. R., \& Costa, J. E. R. 2005, A\&A, 433, 365

Selhorst, C. L., Silva, A. V. R., Costa, J. E. R., \& Shibasaki, K. 2003, A\&A, 401, 1143

Shibasaki, K. 1998, in ASP Conf. Ser., 140, 373

Shibata, K., \& Suematsu, Y. 1982, Sol. Phys., 78, 333

Sterling, A. C. 2000, Sol. Phys., 196, 79

Unruh, Y. C., Solanki, S. K., \& Fligge, M. 1999, A\&A, 345, 635

Zirin, H. 1988 (New York, EUA: Cambridge University Press) 